\title{
Neuroendocrine tumors of the thymus: the oncologist point of view
}

\author{
Nicolas Girard ${ }^{1,2,3}$ \\ ${ }^{1}$ University of Lyon, University Claude Bernard Lyon, Lyon, France; ${ }^{2}$ Department of Respiratory Medicine, National Expert Centre for Thymic \\ Malignancies, Louis Pradel Hospital, Hospices Civils de Lyon, Lyon, France; ${ }^{3}$ Department of Medical Oncology, Institut du Thorax Curie- \\ Montsouris, Institut Curie, Institut Mutualiste Montsouris, Paris, France \\ Correspondence to: Nicolas Girard. Départment d’Oncologie Médicale, Institut Curie, 26 rue d’Ulm, 75248 Paris Cedex 05, France. \\ Email: nicolas.girard2@curie.fr.
}

\begin{abstract}
Thymic malignancies represent a heterogeneous group of rare thoracic cancers, which are classified according to the World Health Organization (WHO) histopathologic classification that distinguishes thymomas from thymic carcinomas and neuroendocrine tumors; neuroendocrine thymic tumors (NETTs) exhibit the same histological spectrum as in other anatomical locations, although with different frequencies. NETTs represent around $2 \%$ of all neuroendocrine tumors, and about $5 \%$ of all thymic malignancies. Overall, the management of patients with NETTs tumors requires continuous multidisciplinary expertise at any step of the disease progression. Systemic treatment relies on cytotoxic chemotherapy, as well as on somatostatin analogues and everolimus. Systemic treatment may be delivered in a curative-intent approach, for patients presenting with locally-advanced tumor at the time of diagnosis, with invasion of intra-thoracic neighboring structures. In such cases, chemotherapy has been used in an induction setting, to reduce the tumor burden-possibly allowing subsequent surgery and/or radiotherapy_or as a postoperative treatment if the tumor was resectable upfront, to reduce the risk of recurrence and achieve prolonged disease control. Systemic therapies are also a palliativeintent treatment of unresectable, metastatic, and recurrent NETTs. Chemotherapy may then be an option for aggressive disease, but somatostatin analogues and everolimus are suitable as well. Alternative options are emerging through clinical trials. As no dedicated study has ever been conducted, recommendations for systemic treatment in NETTs have been mostly based on retrospective cohorts of limited numbers patients, especially in the advanced disease setting, and expert opinion based on experience from primary pulmonary, as well as gastro-intestinal neuroendocrine tumors, for which clinical trials have been conducted.
\end{abstract}

Keywords: Thymoma; thymic carcinoma; network; chemotherapy; radiotherapy; surgery

Submitted Mar 13, 2017. Accepted for publication Jul 14, 2017.

doi: $10.21037 /$ jtd.2017.08.18

View this article at: http://dx.doi.org/10.21037/jtd.2017.08.18

\section{Introduction}

Thymic malignancies represent a heterogeneous group of rare thoracic cancers, which are classified according to the World Health Organization (WHO) histopathologic classification that distinguishes thymomas from thymic carcinomas and neuroendocrine tumors $(1,2)$; neuroendocrine thymic tumors (NETTs) exhibit the same histological spectrum as in other anatomical locations, although with different frequencies. NETTs represent around $2 \%$ of all neuroendocrine tumors, and about $5 \%$ of all thymic malignancies (3). NETTs are characterized by neuroendocrine morphology and differentiation, and comprise carcinoid tumors, which are classified as well-differentiated neuro-endocrine tumors, large cell neuroendocrine carcinoma (LCNEC) and small cell cancer (SCC), which are high-grade, poorly-differentiated tumors (1). Carcinoids are further subdivided into typical (low-grade) and atypical (intermediate grade) carcinoids, based on mitotic count and presence of necrosis (1,2). Meanwhile, the European Neuroendocrine Tumors Society (ENETS) developed a grading system of three groups: G1 (fewer than 2 mitoses per $10 \mathrm{HPFs}$ and/or Ki-67 index of $2 \%$ or less); G2 (2 to 20 mitoses per $10 \mathrm{HPFs}$ and/or Ki-67 index of $3 \%$ to $20 \%$ ); and G3 (more than 20 mitoses per $10 \mathrm{HPFs}$ 
and Ki-67 index higher than 20\%) (4). The ENETS grading was validated as a prognostic variable in NETTs in a series of 28 patients (5). Ultimately, primary location, beyond histology and grade, matters as an independent prognostic variable, as thymic NETTs are more aggressive than primary pulmonary carcinoids, with more frequent invasion and metastatic recurrences $(4,6)$ : carcinoid/low- to intermediate grade NETTs present with a 5-year survival of $50-70 \%$ and a 10 -year survival of only $10-30 \%$ (vs. $90 \%$ and $85 \%$ for lung carcinoids); high-grade NETTs have a 5 -year survival of nearly $0 \%$ (7-14). Recurrence rates after initial treatment is high, ranging from $40 \%$ to $70 \%$ at 5 -year for non-SCC NETTs (13).

Staging of thymic tumors, including NETTs, has historically been based on the Masaoka-Koga system (15), that is being replaced by a tumor nodes metastases (TNM)based system for the 8th Edition of the American Joint Committee on Cancer/Union for International Cancer Control staging classification of tumours (16); the TNM staging better suits NETTs that have a spontaneous propensity towards lymphogeneous and hematological metastases, and, similar to other thymic tumors, provides with a more consistent definition of tumor resectability, which drives the treatment strategy (16).

Diagnosis imaging relies on standard computed tomography (CT) scan, 18-fluorodesoxyglucose position emission tomography (PET)-scan, and more specifically, somatostatin receptor-based imaging, i.e., indium-111 octreoscan or more recently gallium-68 DOTATATE PET/CT if available $(3,6)$; determining the metabolic pattern of the tumor is recommended, as this may be useful for subsequent follow-up and detection of recurrences (17).

A prerequisite before any medical oncology treatment is discussed is to screen patients for endocrine secretion syndromes, the most frequent being carcinoid or Cushing syndrome, which, even if rare (less than $5 \%$ ), may occur, especially in the setting of metastatic disease $(3,6)$. Indeed the delivery of chemotherapy may require hyperhydration that may worsen latent carcinoid syndrome leading to heart failure or severe hypertension; echocardiography should then be carried out at diagnosis (18). Clinical and if needed, genetic assessment for type 1 multiple endocrine neoplasia (MEN1) is also recommended, as follow-up for patients and relatives may be impacted; meanwhile recurrence may not be misdiagnosed with second cancer (19). Lastly, association with myasthenia gravis is unusual, and may lead to review the histological diagnosis of NETTs.

\section{Treatment strategy in NETTs}

The management of NETTs is a paradigm of cooperation between clinicians, surgeons, and pathologists from establishing the diagnosis to organizing the multimodal therapeutic strategy $(3,6,20,21)$. Surgery is the mainstay of the curative-intent treatment, as complete resection represents the most significantly favorable prognostic factor on overall survival (5,7-14).

Systemic treatment relies on cytotoxic chemotherapy, as well as on somatostatin analogues and everolimus. Systemic treatment may be delivered in a curative-intent approach, for patients presenting with locally-advanced tumor at the time of diagnosis, with invasion of intra-thoracic neighboring structures. In such cases, chemotherapy has been used in an induction setting, to reduce the tumor burden-possibly allowing subsequent surgery and/or radiotherapy—or as a postoperative treatment if the tumor was resectable upfront, to reduce the risk of recurrence and achieve prolonged disease control (Figure 1) $(3,6,20,21)$.

Systemic therapies are also a palliative-intent treatment of unresectable, metastatic, and recurrent NETTs (Figure 1) $(3,6,20,21)$. Chemotherapy may then be an option for aggressive disease, but somatostatin analogues and everolimus are suitable as well. Alternative options are emerging through clinical trials.

As no dedicated study has ever been conducted, recommendations for systemic treatment in NETTs have been mostly based on retrospective cohorts of limited numbers patients, especially in the advanced disease setting (5,7-14), and expert opinion based on experience from primary pulmonary, as well as gastro-intestinal neuroendocrine tumors, for which clinical trials have been conducted $(3,6,20,21)$.

\section{Post-operative chemotherapy for NETTs}

Postoperative chemotherapy is not part of the routine treatment strategy for non-neuroendocrine thymic epithelial tumors $(20,22)$; while virtually no data are available for Masaoka-Koga stage I-II thymic epithelial tumors or more advanced thymomas, chemotherapy may still be considered after upfront complete resection of Masaoka-Koga stage III thymic carcinomas, given the high rate of systemic recurrences occurring in more than $30 \%$ of cases (23).

Similarly, as Masaoka-Koga stage III NETTs may present with frequent and early locoregional and/or systemic recurrences after surgery, even if resection is complete 


\section{Neuroendocrine Thymic Tumors}

\begin{tabular}{|l|}
\hline Pre-treatment \\
workup \\
Octreoscan/Dotatate PET-CT \\
scan \\
18 -FDG PET-scan \\
Assessment for MEN1 \\
Endocrine secretion syndrome \\
screening \\
Echocardiography
\end{tabular}

\section{Carcinoid tumors}

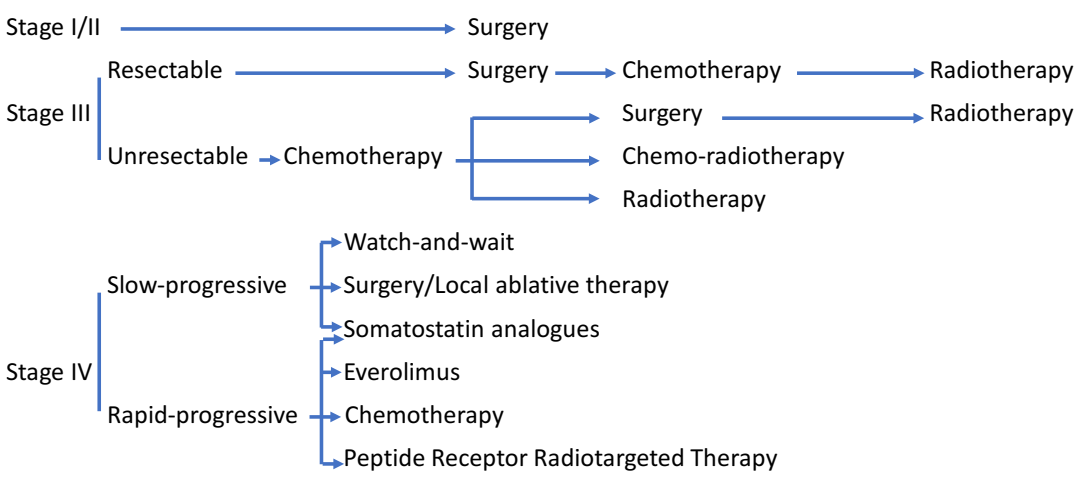

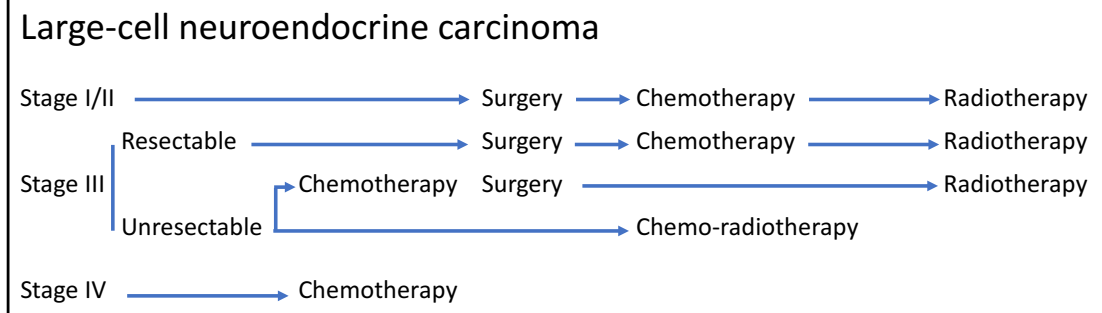

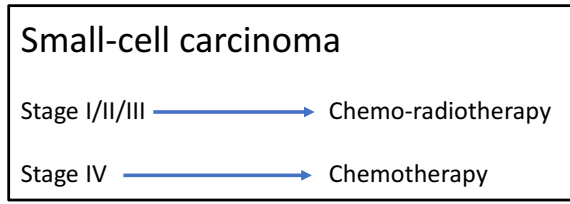

Figure 1 Neuroendocrine thymic tumors. PET, position emission tomography; CT, computed tomography; MEN1, type 1 multiple endocrine neoplasia.

$(13,14)$, postoperative chemotherapy with platin and etoposide may be considered in this setting (Figure 1), if not delivered as induction treatment: whilst the potential benefit seems limited for typical/low-grade carcinoids, given low recurrence rates, postoperative chemotherapy may be discussed after complete resection stage III NETTs, as an option atypical/intermediate grade carcinoids, and as a standard for LCNEC. Still the strategy is debated, for example after complete resection of Masaoka-Koga stage II atypical carcinoid NETTs: in the previous version of the National Comprehensive Cancer Network (NCCN) clinical practice guidelines, postoperative chemotherapy used to be recommended, while in the most recent update, it has been reserved to incomplete resection situations (21); some multidisciplinary teams consider postoperative chemotherapy to cases with a higher proliferative index, or invasion of lymph nodes. In a large series of NETTs, $72 \%$ of which were well-differentiated, postoperative chemotherapy had been delivered to $24 \%$ of patients (14).

With regards to thymic SCC, surgery is usually not part of the treatment strategy, except if diagnosis had not been made in the preoperative setting; similar to what is done in primary bronchopulmonary SCC, post-operative chemotherapy may be recommended for Masaoka-Koga stage II/III tumors $(20,21)$.

The combination of postoperative chemotherapy with mediastinal radiotherapy is systematically proposed in the NCCN guidelines, given the frequent occurrence of locoregional recurrences (Figure 1) (21); this is mostly based on what is now established for thymic carcinomas 
that harbor a similar pattern of recurrences (13), and for which postoperative radiotherapy provides with an overall survival benefit in stage II and III tumors $(20,24)$. In the European series, $52 \%$ of patients had received postoperative radiotherapy (14).

After surgical resection possibly followed by chemotherapy and radiotherapy, the role of consolidation therapy with somatostatin analogues is not established in the absence of endocrine secretion syndrome $(3,6)$.

\section{Preoperative/induction chemotherapy for locally- advanced NETTs}

\section{Preoperative/induction chemotherapy for unresectable locally-advanced NETTS at diagnosis}

Overall, it is estimated that $30 \%$ of patients diagnosed with thymic epithelial tumor present with locally-advanced tumor at time of diagnostic (25). If complete resection is deemed not to be achievable upfront, what is the case in MasaokaKoga stage III/IVA tumors (classified as stage IIIA/IIIB/IVA in the TNM system), after a biopsy is performed, primary/ induction chemotherapy is administered, part of curativeintent sequential strategy integrating subsequent surgery or radiotherapy (Figure 1) (20). Such surgical strategy is usually not proposed for primary thymic SCC then considered as advanced stage and not eligible to focal treatment, similar to what is done in lung SCC.

While preoperative chemotherapy has become standard for locally-advanced, primarily unresectable thymoma and thymic carcinomas (20), virtually no data are available for tumors with NETT with carcinoid histology $(13,14)$; this may indicate: (I) the low frequency of primarily unresectable locally-advanced carcinoid NETT; this may be related to the low number of patients who remain asymptomatic at earlier stage of the disease; (II) the fact that multidisciplinary teams ultimately prefer a strategy of postoperative chemotherapy after upfront resection, what may be related to challenges in making the diagnosis of NETTs on small biopsies.

Given the limited experience from literature data, an expert opinion would be, in the setting of unresectable, non-metastatic NETTs-with carcinoids and LCNEC histology - to treat patients similar to non-neuroendocrine thymic epithelial tumors, with preoperative platinetoposide chemotherapy, aiming at reducing the tumor burden to subsequently allow focal treatment (Figure 1) $(20,22)$. The potential benefit of other chemotherapy regimens or others agents used in the metastatic setting for NETTs is unclear.

Overall, 2-4 cycles may be administered before imaging is performed to reassess the resectability of the tumor. After induction chemotherapy, surgery should be subsequently offered to patients for whom complete resection is thought to be achievable; extended resections may be required $(13,14,26)$. Hyperthermic intrapleural chemotherapy, as well as extra-pleural pneumonectomy may be discussed in case of stage IVA tumor, with no reported case in NETTs, and uncertainly regarding chemotherapy regimens (27). Postoperative radiotherapy is usually delivered afterwards.

Subtotal resection, so called "debulking", may be an option in selected cases of carcinoid NETTs, similar to what may be proposed in thymomas, aiming at facilitating subsequent definitive radiotherapy (28). Debulking is not recommended for poorly-differentiated/LCNEC or SCC NETTs. Postoperative chemoradiotherapy (including additional cycles of cisplatin plus etoposide chemotherapyif response was observed to induction chemotherapy-and a total dose of radiation of $60 \mathrm{~Gy}$ ) may be considered after debulking/R2 resection.

After induction chemotherapy, when the patient is not deemed to be a surgical candidate-either because R0 resection is not thought to be achievable, or because of poor performance status or co-existent medical condition, definitive radiotherapy is recommended part of a chemoradiotherapy strategy $(20,29)$, that may be sequential but possibly concurrent if first assessment is done after the first 2 cycles (Figure 1).

\section{Definitive chemoradiotherapy}

Definitive chemoradiotherapy is the standard of care for non-metastatic, locally-advanced SCC NETTs, and may also be an option for carcinoid or LCNEC NETTs for which surgery is though not to be feasible, even after induction chemotherapy, because of mediastinal or extensive pleural invasion (TNM stage IIIB or IVA) (Figure 1) (29). In a single-arm phase II study with platin and etoposide combined with 45 Gy of thoracic radiotherapy, a complete resection rate of $77 \%$ was reported in non-neuroendocrine thymic epithelial tumors (30).

Ultimately, as in locally-advanced non-small cell lung cancer, the optimal sequence of the multimodal approach is based on response to chemotherapy, level of invasion, and chance of achieving complete resection. Multidisciplinary discussion is mandatory. 


\section{Systemic treatment of metastatic NETTs}

\section{Treatment strategies for metastatic NETTS}

It is estimated that the incidence of metastatic spread in NETTs at the time of diagnosis (Masaoka-Koga and TNM stage IVB) ranges from $20 \%$ in carcinoids to $80 \%$ in SCC NETTs. Systemic treatment may then be offered as a single modality treatment to improve tumor-related symptoms through achievement of tumor stabilization or response, while prolonged survival remains uncertain but may be obtained as NETTs may present with slow growth rate, and as multiple lines and treatment sequences may be applied (22). Given the high frequency of liver metastases, the strategy in NETTs is mostly based on results of trials conducted in gastro-intestinal neuroendocrine tumors. Still, some of those trials also enrolled patients with NETTs. Overall, RECIST v1.1 criteria should be used to assess response to chemotherapy; adapted criteria for pleural lesions include the use of short axis as the measurement plane, and the unidimensional measurement of two pleural tumor sites at three different levels (31).

The first major driver of the systematic treatment strategy in advanced NETTs is histology, as welldifferentiated/carcinoids and poorly differentiated/LCNEC or SCC NETTs will be treated according to different approaches. Other drivers, for well-differentiated/carcinoids are the spontaneous growth rate without treatment, the metastatic spread, and the level of uptake at metabolic imaging $(3,6,21)$. Treatment of endocrine secretion syndrome will not be discussed.

\section{Treatment of metastatic poorly-differentiated NETTs: LCNEC and SCC}

The treatment strategy relies on a similar approach to nonneuroendocrine thymic epithelial tumors (20). Cisplatinbased combination regimens with anthracyclins and/ or more likely etoposide are standard (Figure 1) (32-34). No randomized studies have been conducted, and it is unclear which regimens are best; multi-agent combination regimens and anthracycline-based regimens appear to have improved response rates compared to others, especially the etoposide, ifosfamide and cisplatin combination, in thymic epithelial tumors overall. Combination of carboplatin and paclitaxel is an option, based on results of phase II trials that enrolled a few NETTs patients (35-37). Response rates range from $30 \%$ to $50 \%$, with progression-free survival rates ranging from 6 to 9 months (22).

\section{Treatment of metastatic well-differentiated NETTs: typical and atypical carcinoids}

In metastatic carcinoid NETTs, the prognosis heterogeneity and the absence of curative treatment make quality of life a major endpoint of the treatment strategy. Compared to other anatomical location of neuroendocrine tumors, NETTs are associated with a higher number of patients with distant metastases at diagnosis, a higher prevalence of bone, liver metastases, and a lower rate of endocrine secretion syndromes. Multiple approaches are possible, based on histology, tumor burden and growth rate (Figure 1).

\section{Watch-and-wait approach}

In asymptomatic patients, with a low tumor burden, slowlyand non-progressive disease and without threatening metastases, a watch and see policy might be considered and explained to the patients on the basis of regular CT imaging every 2 to 6 months $(3,6)$.

\section{Surgery and local-ablative approaches}

Surgery and/or local-ablative approaches may be considered for patients with a limited number of metastatic sites, all amenable to radical treatment, in the setting of slowly progressive disease, with a "curative" intent $(3,6)$. Surgery, as well as stereotactic radiotherapy, or percutaneous procedures such as radiofrequency or cryoablation may be used. Surgical resection of liver metastases can be considered in patients with no extra-abdominal metastases, no lymph node invasion or peritoneal carcinomatosis. Complete resection of liver metastases in gastro-intestinal tumors was associated with prolonged survival, above $70 \%$ at 5 -year (38). Selective embolization with particles (transarterial embolization, TAE) or cytotoxics such as doxorubicin (TACE) may be effective whenever surgical resection is not feasible (38).

\section{Somatostatin analogues}

Somatostatin analogues are standard treatment both for slowly- and actively-progressive tumors, after the results of two landmark clinical trials, PROMID and CLARINET, that enrolled patients with gastro-intestinal neuroendocrine tumors to received octreotide LAR ( $30 \mathrm{mg} / 28$ days) or lanreotide Autogel (120 mg/28 days) vs. placebo $(39,40)$. In PROMID, $40 \%$ of patients had endocrine secretion syndromes, when CLARINET focused on non-functional neuroendocrine tumors. Octreoscan imaging was positive in $75 \%$ of cases in PROMID, and $100 \%$ of cases in 
CLARINET. Results of those trials indicates a benefit of somatostatin analogues, with median time to progression significantly of 14 months versus 6 months with placebo in PROMID (39), and $62 \%$ vs. $22 \%$ of non-progressive patients after 8 years of follow-up in CLARINET (40). SPINET is a phase III trial dedicated to the management of thoracic neuroendocrine tumors, enrolling patients with primary pulmonary and thymic carcinoid NETTs, and assessing lanreotide autogel $v s$. placebo in this setting (Clinicaltrials.gov ID NCT02683941).

\section{Everolimus}

Everolimus is becoming a therapeutic standard after the failure of other treatments in typical and atypical carcinoids. RADIANT-2 was a randomized trial that compared everolimus $10 \mathrm{mg} /$ day plus octreotide $v s$. placebo + octreotide in more than 400 patients with non-pancreatic functioning (carcinoid syndrome) neuroendocrine tumors. The study demonstrated a clinically significant 5.1 months increase in median progression-free survival in the everolimus arm (41). RADIANT-4 compared everolimus $10 \mathrm{mg}$ to placebo in non-functioning, progressive lung and gastrointestinal neuroendocrine tumors, and demonstrated a median progression-free survival of 11.0 months in the everolimus group, vs. 3.9 months in the placebo group [hazard ratio (HR) 0.48 (95\% CI, 0.35-0.67)] (42); the survival benefit was similar in all anatomical locations; overall survival analysis was also significantly higher in the everolimus arm [HR 0.64 (95\% CI, 0.40-1.05)].

The ongoing LUNA study is a three-arm phase II trial assessing everolimus alone $v s$. pasireotide $v s$. the combination of everolimus plus pasireotide, and has completed its recruitment (NCT01563354); primary objective is the proportion of patients with overall lesion assessment at month 9 being response or stable disease according to RECIST.

Meanwhile, everolimus is now approved for the treatment of well-differentiated, progressive gastro-intestinal and pulmonary NETTs in Europe. The challenge is now to optimally select patients: Is everolimus as second-line treatment after somatostatin analogues, or a first-line treatment before somatostatin analogues or chemotherapy in non-functioning tumors? Which growth rate is relevant for each of those strategies? What is the safety in a realpractice setting?

\section{Cytotoxic chemotherapy}

Systemic cytotoxic chemotherapy is an option in patients with advanced, unresectable, progressive well-differentiated
NETTs. In general, regimens are similar to that described for poorly-differentiated tumors, with platin and etoposide combination being the preferred option among thoracic oncology teams $(20,21)$. A $20 \%$ response rate to cisplatin or carboplatin was reported in two studies specifically dedicated to well-differentiated lung carcinoids (43).

Alternative regimens have been used, mostly in gastrointestinal neuroendocrine tumors, including 5 -fluorouracil (5-FU), dacarbazine, and temozolomide (TMZ) alone or in combination (44-46). TMZ may represents a first-line option for cytotoxic chemotherapy for patients with wellor moderately differentiated NETT, because of fewer side effects $(47,48)$.

Alternative options include 5-FU-based regimens: a retrospective analysis of 5-FU-STZ and cisplatin that included 79 patients with progressive neuroendocrine tumors reported a response rate for non-pancreatic primary sites of $25 \%$, with overall median progression-free survival of 9.1 months (44). Combination of gemcitabine or 5 -FU plus oxaliplatine produced disease control rates of in a recent retrospective analysis (49).

\section{Peptide receptor radiotargeted therapy (PRRT)}

In octreoscan or Gallium-68 dotatate PET scanpositive tumors, PRRT may be used to treat metastatic carcinoids, with currently 90Yttrium-DOTA octreotide and 177-Lutetium DOTA octreotide being the most used regimens in selected patients. Although most studies are limited to single centers, a large retrospective study looking at 1,109 metastatic neuroendocrine tumors, including 84 lung carcinoids, response rate was $28 \%$ with a mean survival of 40 months. Grade 3-4 toxicity is reported in $10-33 \%$ of patients, mainly renal or hematological toxicities including irreversible renal toxicity (9\%) (50).

\section{Antiangiogenics}

While sunitinib is considered an option in advanced, refractory non-neuroendoendocrine thymic epithelial tumors (21), the interest of antiangiogenic agents in NETTs remains uncertain. A phase II study evaluated the activity of sunitinib in 109 patients with metastatic neuroendocrine tumors, including 41 carcinoids; disease control was achieved in $85 \%$ in those patients, with a progression-free survival of 10 months (51). Similar results were reported with pazopanib (52). Phase II studies also evaluated bevacizumab alone or in combination with sorafenib (53). Then the off-label use of antiangiogenics has to be reserved to refractory NETTs, after systematic discussion at an 
expert multidisciplinary tumor board.

\section{Recurrent NETTs}

NETTs are more aggressive than primary pulmonary or gastro-intestinal neuroendocrine tumors. Also, amongst thymic tumors, NETTs are associated with the lowest survival rate and the highest rate of local-regional, lymph node, and distant metastases. Five-year survival rates after treatment of NETT vary considerably, from as low as $28 \%$ to $84 \%(5,7-14)$. Such variability may reflect the different accrual of the centers, as well as the evolving management strategies over the years.

The management of recurrences of NETTs follows the same strategy as primary tumors after the initial diagnosis. The extent of the recurrence should carefully be evaluated using imaging techniques, including metabolic scintigraphy. Histologic confirmation of recurrence may be required.

Treatment of recurrence largely depends on its extent $(3,6,21)$. Repeated resection may be considered for local or regional recurrence, with reported satisfactory long-term survival. In well-differentiated/carcinoid tumors, surgicallyresectable lesions may be operated on upfront, while local-ablative therapies may also be applied; postoperative strategy may include somatostatine analogues, or even chemotherapy in rapidly-progressive disease. In the setting of systemic recurrences, management relies on multiple lines of treatment, including somatostatin analogues, everolimus, chemotherapy regimens, and PRRT, as described above for upfront metastatic tumors.

In LCNEC and SCC, multiple lines of chemotherapy may be administered as well. Ongoing phase I/II or even phase III trials are evaluating innovative treatments, such as immunotherapy with anti-PD1/PD-L1, anti-CTLA4, antiDLL3 antibodies, or other investigational agents.

In non-resectable recurrences, several consecutive lines of chemotherapy may be administered when the patient presents with tumor progression. The re-administration of a previously effective regimen has to be considered, especially in case of previous response, late occurring recurrence.

\section{Conclusions}

Overall, the management of patients with NETTs tumors requires continuous multidisciplinary expertise at any step of the disease progression. A dramatic improvement in our knowledge of the management of thymic tumors has occurred in the last few years, resulting in the development of databases, translational research programs, and clinical trials. While access to innovative strategies represents a major challenge, as the rarity of the tumor precludes specific approval of drugs to be obtained, patientcentered initiatives, such as the establishment of dedicated networks, are warranted. In France, RYTHMIC (Réseau tumeurs THYMiques et Cancer) is a nationwide network for thymic malignancies, which was appointed in 2012 by the French National Cancer Institute, as part of its rare cancer program. Since then, the management of all patients diagnosed with thymic tumors has been discussed on a real-time basis at a national multidisciplinary tumor board, which is organized twice a month basis using a web-based conferencing system. A prospective database of all patients is hosted by the French Thoracic Cancer Intergroup that provides a unique resource to gain insight in the results of systemic treatment. Interestingly a similar network has been appointed for neuroendocrine tumors, which is called RENATEN, allowing multidisciplinary discussion with neuroendocrine tumor specialists as well. At the European Union level, the recent approval of the European Reference Network EURACAN, dedicated to the care of patients with rare cancers, including neuroendocrine tumors and thymic tumors, aims at ensuring patients an equal access to highly specialized management, and will provide with a comprehensive tools to monitor dedicated actions to improve the management of complex cancers, NETTs being a typical situation requiring multidisciplinary approaches and research.

\section{Acknowledgements}

None.

\section{Footnote}

Conflicts of Interest: Nicolas Girard has reported consultancy from BMS, Eli-Lilly, Novartis, Pfizer.

\section{References}

1. WHO histological classification of tumours of the thymus. In: Travis WB, Brambilla A, Burke AP, et al. World Health Organization Classification of Tumours of the Lung, Pleura, Thymus and Heart. Lyon: IARC Press; 2015.

2. Marx A, Ströbel P, Badve SS, et al. ITMIG consensus statement on the use of the WHO histological classification of thymoma and thymic carcinoma: refined 
definitions, histological criteria, and reporting. J Thorac Oncol 2014;9:596-611.

3. Öberg K, Hellman P, Ferolla P, et al. ESMO Guidelines Working Group. Neuroendocrine bronchial and thymic tumors: ESMO Clinical Practice Guidelines for diagnosis, treatment and follow-up. Ann Oncol 2012;23 Suppl 7:vii120-3.

4. Rindi G, Klöppel G, Couvelard A, et al. TNM staging of mid gut and hindgut (neuro) endocrine tumors: a consensus proposal including a grading system. Virchows Arch 2007;451:757-62.

5. Crona J, Björklund P, Welin S, et al. Treatment, prognostic markers and survival in thymic neuroendocrine tumours. a study from a single tertiary referral centre. Lung Cancer 2013;79:289-93.

6. Caplin ME, Baudin E, Ferolla P, et al. ENETS consensus conference participants. Pulmonary neuroendocrine (carcinoid) tumors: European Neuroendocrine Tumor Society expert consensus and recommendations for best practice for typical and atypical pulmonary carcinoids. Ann Oncol 2015;26:1604-20.

7. Fukai I, Masaoka A, Fujii Y, et al. Thymic neuroendocrine tumor (thymic carcinoid): a clinicopathologic study in 15 patients. Ann Thorac Surg 1999;67:208-11.

8. Moran CA, Suster S. Neuroendocrine carcinomas (carcinoid tumor) of the thymus. a clinicopathologic analysis of 80 cases. Am J Clin Pathol 2000;114:100-10.

9. Tiffet O, Nicholson AG, Ladas G, et al. A clinicopathologic study of 12 neuroendocrine tumors arising in the thymus. Chest 2003;124:141-6.

10. Gaur P, Leary C, Yao JC. Thymic neuroendocrine tumors: a SEER da- tabase analysis of 160 patients. Ann Surg 2010;251:1117-21.

11. Ahn S, Lee JJ, Ha SY, et al. Clinicopathological analysis of 21 thymic neuroendocrine tumors. Korean J Pathol 2012;46:221-5.

12. Cardillo G, Rea F, Lucchi M, et al. Primary neuroendocrine tumors of the thymus: a multicenter experience of 35 patients. Ann Thorac Surg 2012;94:241-5.

13. Filosso PL, Yao X, Ruffini E, et al. Comparison of outcomes between neuroendocrine thymic tumours and other subtypes of thymic carcinomas: a joint analysis of the European Society of Thoracic Surgeons and the International Thymic Malignancy Interest Group. Eur J Cardiothorac Surg 2016:50:766-71.

14. Filosso PL, Yao X, Ahmad U, et al. Outcome of primary neuroendocrine tumors of the thymus: a joint analysis of the International Thymic Malignancy Interest Group and the European Society of Thoracic Surgeons databases. J
Thorac Cardiovasc Surg 2015;149:103-9.e2.

15. Detterbeck FC, Nicholson AG, Kondo K, et al. The Masaoka-Koga Stage Classification for Thymic Malignancies: Clarification and Definition of Terms. J Thorac Oncol 2011;6:S1710-6.

16. Detterbeck FC, Stratton K, Giroux D, et al. The IASLC/ ITMIG Thymic Epithelial Tumors Staging Project: proposal for an evidence-based stage classification system for the forthcoming (8th) edition of the TNM classification of malignant tumors. J Thorac Oncol 2014;9:S65-72.

17. Ruf J, Schiefer J, Furth C, et al. 68Ga-DOTATOC PET/ CT of neuroendocrine tumors: spotlight on the CT phases of a triple-phase protocol. J Nucl Med 2011;52:697-704.

18. Plöckinger U, Gustafsson B, Ivan D, et al. ENETS Consensus Guidelines for the Standards of Care in Neuroendocrine Tumors: echocardiography. Neuroendocrinology 2009;90:190-3.

19. Thakker RV, Newey PJ, Walls GV, et al. Clinical practice guidelines for multiple endocrine neoplasia type 1 (MEN1). J Clin Endocrinol Metab 2012;97:2990-3011.

20. Girard N, Ruffini E, Marx A, et al. Thymic epithelial tumours: ESMO Clinical Practice Guidelines for diagnosis, treatment and follow-up. Ann Oncol 2015;26 Suppl 5:v40-55.

21. Available online: https://www.nccn.org/professionals/ physician_gls/pdf/neuroendocrine.pdf

22. Girard N, Lal R, Wakelee H, et al. Chemotherapy definitions and policies for thymic malignancies. J Thorac Oncol 2011;6:S1749-55.

23. Detterbeck F. Towards a TNM based prognostic classification for thymic tumours. J Thorac Oncol 2013;8:S68.

24. Rimner A, Yao X, Huang J, et al. Postoperative Radiation Therapy Is Associated with Longer Overall Survival in Completely Resected Stage II and III ThymomaAn Analysis of the International Thymic Malignancies Interest Group Retrospective Database. J Thorac Oncol 2016;11:1785-92.

25. Ruffini E, Detterbeck F, Van Raemdonck D, et al. Tumours of the thymus: a cohort study of prognostic factors from the European Society of Thoracic Surgeons database. Eur J Cardiothorac Surg 2014;46:361-8.

26. Wright CD. Extended resections for thymic malignancies. J Thorac Oncol 2010;5:S344-7.

27. Yellin A, Simansky DA, Ben-Avi R, et al. Resection and heated pleural chemoperfusion in patients with thymic epithelial malignant disease and pleural spread: a single-institution experience. J Thorac Cardiovasc Surg 
2013;145:83-7.

28. Hamaji M, Kojima F, Omasa M, et al. A meta-analysis of debulking surgery versus surgical biopsy for unresectable thymoma. Eur J Cardiothorac Surg 2015;47:602-7.

29. Loehrer PJ Sr, Chen M, Kim K, et al. Cisplatin, doxorubicin, and cyclophosphamide plus thoracic radiation therapy for limited-stage unresectable thymoma: an intergroup trial. J Clin Oncol 1997;15:3093-9.

30. Korst RJ, Bezjak A, Blackmon S, et al. Neoadjuvant chemoradiotherapy for locally advanced thymic tumors: a phase II, multi-institutional clinical trial. J Thorac Cardiovasc Surg 2014;147:36-44.

31. Huang J, Detterbeck FC, Wang Z, et al. Standard outcome measures for thymic malignancies. J Thorac Oncol 2011;6:S1691-7.

32. Loehrer PJ Sr, Kim K, Aisner SC, et al. Cisplatin plus doxorubicin plus cyclophosphamide in metastatic or recurrent thymoma: final results of an intergroup trial. The Eastern Cooperative Oncology Group, Southwest Oncology Group, and Southeastern Cancer Study Group. J Clin Oncol 1994;12:1164-8.

33. Giaccone G, Ardizzoni A, Kirkpatrick A, et al. Cisplatin and etoposide combination chemotherapy for locally advanced or metastatic thymoma. A phase II study of the European Organization for Research and Treatment of Cancer Lung Cancer Cooperative Group. J Clin Oncol 1996;14:814-20.

34. Loehrer PJ Sr, Jiroutek M, Aisner S, et al. Combined etoposide, ifosfamide, and cisplatin in the treatment of patients with advanced thymoma and thymic carcinoma: an intergroup trial. Cancer 2001;91:2010-5.

35. Igawa S, Yanagisawa N, Niwa H, et al. Successful chemotherapy with carboplatin and nab-paclitaxel for thymic large cell neuroendocrine carcinoma: A case report. Oncol Lett 2015;10:3519-22.

36. Hirai F, Yamanaka T, Taguchi K, et al. A multicenter phase II study of carboplatin and paclitaxel for advanced thymic carcinoma: WJOG4207L. Ann Oncol 2015;26:363-8.

37. Lemma GL, Lee JW, Aisner SC, et al. Phase II study of carboplatin and paclitaxel in advanced thymoma and thymic carcinoma. J Clin Oncol 2011;29:2060-5.

38. Pavel M, Baudin E, Couvelard A, et al. ENETS Consensus Guidelines for the management of patients with liver and other distant metastases from neuroendocrine neoplasms of foregut, midgut, hindgut, and unknown primary. Neuroendocrinology 2012;95:157-76.

39. Rinke A, Müller HH, Schade-Brittinger C, et al. Placebocontrolled, double-blind, prospective, randomized study on the effect of octreotide LAR in the control of tumor growth in patients with metastatic neuroendocrine midgut tumors: a report from the PROMID Study Group. J Clin Oncol 2009;27:4656-63.

40. Caplin ME, Pavel M, wikła JB, et al. Lanreotide in metastatic enteropancreatic neuroendocrine tumors. $\mathrm{N}$ Engl J Med 2014;371:224-33.

41. Pavel ME, Hainsworth JD, Baudin E, et al. Everolimus plus octreotide long-acting repeatable for the treatment of advanced neuroendocrine tumours associated with carcinoid syndrome (RADIANT-2): a randomised, placebocontrolled, phase 3 study. Lancet 2011;378:2005-12.

42. Yao JC, Fazio N, Singh S, et al. Everolimus for the treatment of advanced, non-functional neuroendocrine tumours of the lung or gastrointestinal tract (RADIANT-4): a randomised, placebo-controlled, phase 3 study. Lancet 2016;387:968-77.

43. Wirth LJ, Carter MR, Jänne PA, et al. Outcome of patients with pulmonary carcinoid tumors receiving chemotherapy or chemoradiotherapy. Lung Cancer 2004;44:213-20.

44. Sun W, Lipsitz S, Catalano P, et al. Phase II/III study of doxorubicin with fluorouracil compared with streptozocin with fluorouracil or dacarbazine in the treatment of advanced carcinoid tumors: Eastern Cooperative Oncology Group Study E1281. J Clin Oncol 2005;23:4897-904.

45. Brizzi MP, Berruti A, Ferrero A, et al. Continuous 5-fluorouracil infusion plus long acting octreotide in advanced well-differentiated neuroendocrine carcinomas. A phase II trial of the Piemonte oncology network. BMC Cancer 2009;9:388.

46. Bajetta E, Catena L, Procopio G, et al. Are capecitabine and oxaliplatin (XELOX) suitable treatments for progressing low-grade and high-grade neuroendocrine tumors? Cancer Chemother Pharmacol 2007;59:637-42.

47. Ekeblad S, Sundin A, Janson ET, et al. Temozolomide as monotherapy is effective in treatment of advanced malignant neuroendocrine tumors. Clin Cancer Res 2007;13:2986-91.

48. Chan JA, Stuart K, Earle CC, et al. Prospective study of bevacizumab plus temozolomide in patients with advanced neuroendocrine tumors. J Clin Oncol 2012;30:2963-8.

49. Walter T, Planchard D, Bouledrak K, et al. Evaluation of the combination of oxaliplatin and 5-fluorouracil or gemcitabine in patients with sporadic metastatic pulmonary carcinoid tumors. Lung Cancer 2016;96:68-73.

50. Imhof A, Brunner P, Marincek N, et al. Response, survival, and long-term toxicity after therapy with the radiolabeled somatostatin analogue (90Y-DOTA)-TOC in metastasized 
neuroendocrine cancers. J Clin Oncol 2011;29:2416-23.

51. Kulke MH, Lenz HJ, Meropol NJ, et al. Activity of sunitinib in patients with advanced neuroendocrine tumors. J Clin Oncol 2008;26:3403-10.

52. Grande Pulido E, Castellano DE, Rocio GarciaCarboneroR, et al. PAZONET: Results of a phase II trial of pazopanib as a sequencing treatment in progressive metastatic neuroendocrine tumors (NETs) patients (pts), on behalf of the Spanish task force for NETs (GETNE) NCT01280201. J Clin Oncol 2012:30:abstr 4119.

53. Castellano D, Capdevila J, Sastre J, et al. Sorafenib and bevacizumab combination targeted therapy in advanced neuroendocrine tumour: a phase II study of Spanish Neuroendocrine Tumour Group (GETNE0801). Eur J Cancer 2013;49:3780-7.
Cite this article as: Girard N. Neuroendocrine tumors of the thymus: the oncologist point of view. J Thorac Dis 2017;9(Suppl 15):S1491-S1500. doi:10.21037/jtd.2017.08.18 